\title{
Translational Diffusion in Polydisperse Polymer Samples Studied by Dynamic Imaging of Diffusion ESR
}

\author{
Dajiang Xu, Elizabeth Hall, ${ }^{\dagger, \$}$ Christopher K. Ober, $\$$ Jozef K. Moscicki, ${ }^{\S}$ and Jack H. Freed $*$ \\ Baker Laboratory of Chemistry, Cornell University, Ithaca, New York 14853-1301
}

Received: February 16, 1996; In Final Form: May 20, $1996^{\otimes}$

\begin{abstract}
A new methodology utilizing the DID-ESR (dynamic imaging of diffusion by ESR) technique has been developed for its application to polydisperse polymer samples. Due to polydispersity the spin-labeled polymer molecules have a wide range of molecular weight, and this is also true for the matrix polymer molecules. This generally leads to a wide distribution in diffusion coefficients of the spin-labeled molecules. The work presented here includes a theoretical derivation of the new DID-ESR method in the presence of polydispersity, plus its first application for measuring translational diffusion coefficients of liquid crystalline (LC) polymer melts. This includes a detailed analysis of the reliability of the method, the proper interpretation of average diffusion coefficients, and how the molecular weight dependence of the diffusion coefficient may be obtained from even a single experiment on a sample with wide polydispersity. The results obtained by this method are compared with those from the FRES (forward recoil spectroscopy) technique on the same model system, but for higher molecular weight, and account is taken of the differences in the two methods. Future experiments to further study LC polymer diffusion mechanisms are proposed.
\end{abstract}

\section{Introduction}

There have been numerous techniques developed to measure translational diffusion coefficients and to verify the theories to predict the diffusional behavior in various liquid crystal (LC) and polymer systems. The primary advantage of the dynamic imaging of diffusion (DID)-ESR technique is that one can simultaneously take conventional CW-ESR spectra and analyze them by ESR spectral simulation to obtain microscopic dynamic information about the same sample. In this way, macroscopic and microscopic properties of the sample can be related to a significant degree.

Unlike the case for a monomeric LC, which exhibits a single diffusion coefficient, for which measurement by DID-ESR is well described in several papers, ${ }^{1-4}$ many polymer samples are polydisperse with respect to the molecular weight, as shown in Figure 1, and the distribution of molecular weights results in a distribution of the diffusion coefficients.

The ultimate goal of the present work is to develop a reliable and efficient technique to measure diffusion coefficients of polydisperse polymer samples. This should enable one to study diffusion mechanisms by observing the dependence of the diffusion coefficient on the molecular weight, the temperature, and the ordering of the polymer samples.

There are several theoretical models to describe the molecular weight effect on polymer diffusion. ${ }^{5}$ In general, the diffusion coefficient $D$ is expected to depend on the molecular weights of the tracer $(M)$ and the matrix $(P)$ according to ${ }^{6}$

$$
D=D_{0} M^{-\alpha} P^{-\beta} \quad(\alpha>0, \beta \geq 0)
$$

where $\alpha, \beta$ are the constants to be determined and $D_{0}$ is a function of the other variables that also affect the diffusion coefficient, such as temperature and ordering. Here we assume

\footnotetext{
$\dagger$ Currently at AT\&T Bell Laboratories, Whippany, NJ 07981-0903.

\$ Dept. of Materials Science and Engineering, Cornell University, Ithaca, NY 14853-1501.

$\S$ On leave from Institute of Physics, Jagiellonian University, Krakow, Poland.

${ }^{\otimes}$ Abstract published in Advance ACS Abstracts, September 15, 1996.
}

\section{Molecular Weight Distribution}
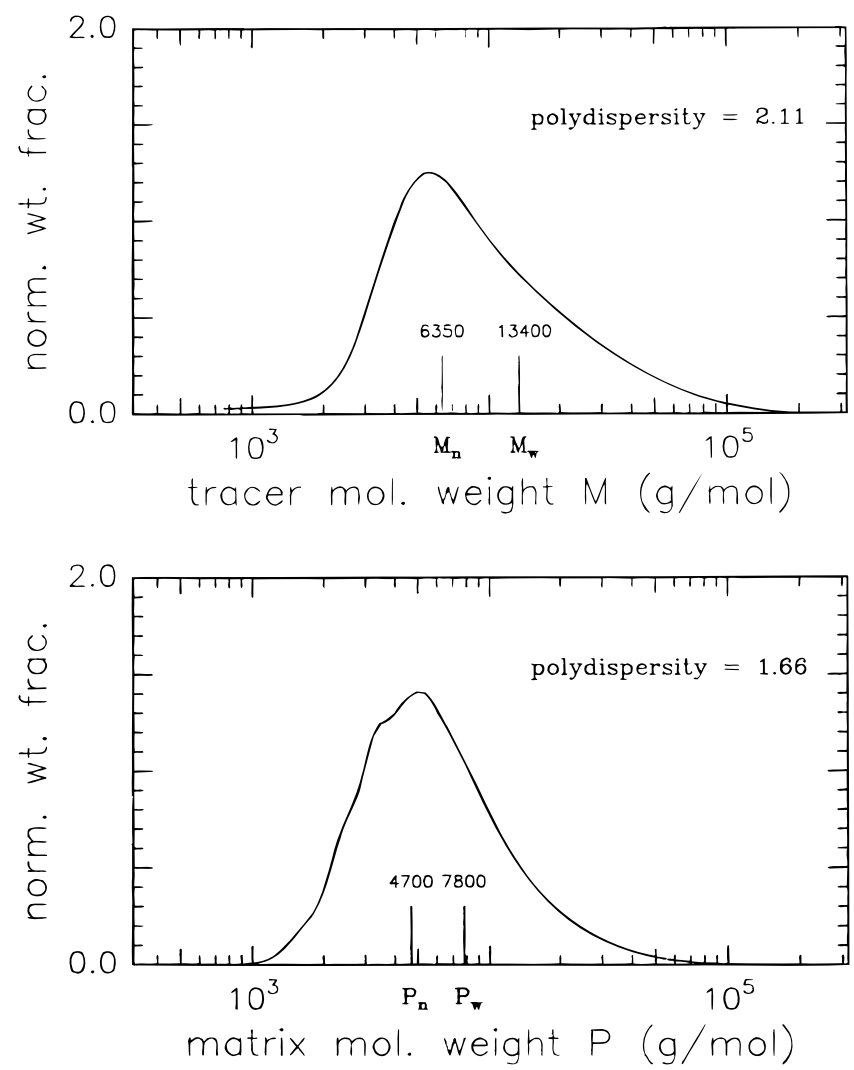

Figure 1. Molecular weight distribution functions of spin-labeled DHMS-7,9 (tracer $C$ ) and of unlabeled DHMS-7,9 (matrix $E$ ) (cf. Table $1)$.

that $D_{0}$ is independent of the molecular weights of both tracer and matrix. Typical theoretical models that have been considered in the past ${ }^{5,6}$ include (a) the Rouse model, appropriate for short noninteracting coils, which yields $\alpha=1, \beta=0$; (b) the reptation model, appropriate for entangled chains with $P>M$, 
Diffusion Coefficient Distribution
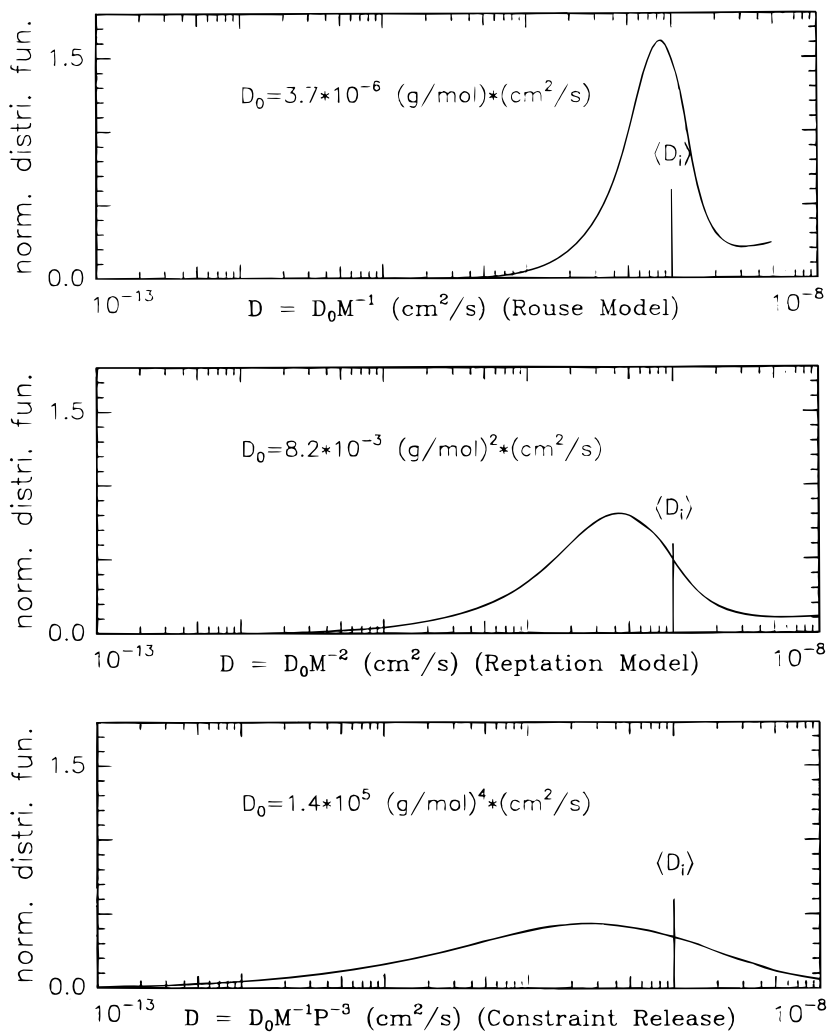

Figure 2. Diffusion coefficient distribution functions based on the molecular weight distributions of Figure 1 and some polymer diffusion models: (a) Rouse model; (b) reptation model; (c) constraint release model (this assumes case 2 of the matrix polydispersity effect in section 2.2).

which yields $\alpha=2, \beta=0$; and (c) the constraint release model, appropriate for noninteracting chains with $M \gg P$, which yields $\alpha=1, \beta=3$.

In Figure 2 we plot the predicted distributions of diffusion coefficients resulting from the molecular weight distributions shown in Figure 1 that are based on some of the diffusion models. There is a significant difference in these distributions among the various diffusion models, even from samples with moderate polydispersity. The appropriate interpretation of the measured diffusion coefficient is crucial to the study of its dependence on these other factors, which is needed to better understand the mechanism in LC polymer diffusion.

This paper consists of six sections. In section 2 a theoretical description of the DID-ESR method in the presence of polydispersity is derived. We first define the appropriate average diffusion coefficient, $\left\langle D_{i}\right\rangle$. Then we show how to obtain $\left\langle D_{i}\right\rangle$ from the ESR measurements. We also show how the molecular weight dependence of the diffusion coefficients (i.e., the values of $\alpha, \beta$ in eq 1) are obtained from measurements on several different samples or from a single experiment on a single sample with large polydispersity. We also discuss the sensitivity and reliability of the method. ${ }^{7}$

We chose to work with a semirigid main chain LC polyether with which we had considerable previous experience. ${ }^{6}$ The synthesis and the sample preparation for the DID-ESR measurement are discussed in section 3. A model polymer with the same structure used in previous studies by forward recoil spectroscopy (FRES) ${ }^{6}$ was spin labeled and employed here. Our intention was to study samples with lower molecular weights than could be studied by the FRES method. A successful method of preparation and minor equipment modification enabled measurements to be made at higher temperature and for longer times than previous DID-ESR experiments on ordinary LCs.

In section 4 , we report the experimental results ${ }^{8}$ and illustrate the data analysis procedures utilizing the new methodology described in section 2 .

We compare our results with those obtained by the FRES experiments from the same model system in section 5. Future experiments to further study LC polymer diffusion mechanisms are proposed in that section.

A summary of this work is presented in section 6 .

\section{Theoretical Background}

2.1. Diffusion in Polydisperse Polymer Samples. When there is a distribution of diffusion coefficients in the polymer system being studied, the individual $D$ for each component species is difficult to measure directly with any technique currently available. This is to be contrasted with a distribution in the molecular weights, which can be readily measured for instance by gel permeation chromatography (GPC). Instead, the usual approach is to find some macroscopically observable quantities, e.g., an average $\left\langle D_{i}\right\rangle$, as well as $D_{0}, \alpha, \beta$ in eq 1 .

Suppose each species $i$ has a probability weight of $Y(i),(Y(i)$ is normalized, and we shall relate it to the distribution functions of the tracer and matrix later). Its distinct diffusion coefficient $D_{i}$, expressed by eq 1 , satisfies Fick's second law of diffusion: ${ }^{9 a}$

$$
\frac{\partial C(x, t)}{\partial t}=D_{x} \frac{\partial^{2} C(x, t)}{\partial x^{2}}
$$

where $C_{i}(x, t)$ is the tracer concentration of species $i$, as a function of spatial coordinate $x$ and time $t$. We limit our discussion to one-dimensional diffusion in this paper. In the case of unrestricted diffusion (no boundaries at finite distances), the solution to this homogeneous diffusion equation is

$$
C_{i}(x, t)=\int_{-\infty}^{+\infty} C_{i}\left(x^{\prime}, t_{0}\right) \frac{1}{\sqrt{4 \pi D_{i}\left(t-t_{0}\right)}} \exp \left[-\frac{\left(x-x^{\prime}\right)^{2}}{4 D_{i}\left(t-t_{0}\right)}\right] \mathrm{d} x^{\prime}
$$

where $C_{i}\left(x, t_{0}\right)$ is the initial tracer concentration profile from species $i$ at the initial time $t=t_{0}$. The total tracer concentration profile is

$$
C(x, t)=\int_{i} C_{i}(x, t) \mathrm{d} i
$$

In the case $t_{0}=0$, eq 3 is simplified to

$$
C_{i}(x, t)=\int_{-\infty}^{+\infty} C_{i}\left(x^{\prime}, 0\right) \frac{1}{\sqrt{4 \pi D_{i} t}} \exp \left[-\frac{\left(x-x^{\prime}\right)^{2}}{4 D_{i} t}\right] \mathrm{d} x^{\prime}
$$

Furthermore, we shall make an assumption that at $t=0$ all the species have the same spatial distribution. Thus we can introduce a function $C_{0}(x, 0)$, which is independent of species $i$, such that

$$
C_{i}(x, 0)=Y(i) C_{0}(x, 0)
$$

Then from eq 4, we obtain

$$
C(x, 0)=\int_{i} C_{0}(x, 0) Y(i) \mathrm{d} i=C_{0}(x, 0)
$$

This assumption should be appropriate just after a DID-ESR sample is prepared with its initial inhomogeneous concentration 
distribution. It is a crucial step to convert the microscopic quantity, $C_{i}(x, 0)$, which is very difficult to measure, into an easily measurable macroscopic quantity, $C(x, 0)$.

Substituting eqs 6 and 7 into eq 5 , we obtain

$$
C_{i}(x, t)=Y(i) \int_{-\infty}^{+\infty} C\left(x,{ }^{\prime} 0\right) \frac{1}{\sqrt{4 \pi D_{i} t}} \exp \left[-\frac{\left(x-x^{\prime}\right)^{2}}{4 D_{i} t}\right] \mathrm{d} x^{\prime}
$$

Now substituting eq 8 into eq 4 , exchanging the order of integration, one has

$$
\begin{aligned}
& C(x, t)=\int_{-\infty}^{+\infty} C\left(x^{\prime}, 0\right)\left\{\int_{i} \frac{Y(i)}{\sqrt{4 \pi D_{i} t}} \exp \left[-\frac{\left(x-x^{\prime}\right)^{2}}{4 D_{i} t}\right] \mathrm{d} i\right\} \mathrm{d} x^{\prime} \\
& \text { i.e., } \quad C(x, t)=C(x, 0) \otimes \int_{i} \frac{1}{\sqrt{4 \pi D_{i} t}} \exp \left(-\frac{x^{2}}{4 D_{i} t}\right) Y(i) \mathrm{d} i
\end{aligned}
$$

where the symbol $\otimes$ implies a convolution. Equation 10 can be further simplified after taking a Fourier transform over the spatial coordinate $x$ :

$$
\frac{\tilde{C}(\kappa, t)}{\tilde{C}(\kappa, 0)}=\int_{i} \exp \left[-(2 \pi \kappa)^{2} t D_{i}\right] Y(i) \mathrm{d} i
$$

where $\tilde{C}(\kappa, t)$ is the Fourier transform of the function $C(x, t)$ and $\kappa$ is in units of inverse distance.

Equation 11 is a general expression. The derivation so far is independent of the details of the diffusion process and the molecular weight distributions, even the particular technique. Notice that the right-hand side (rhs) of eq 11 is a quantity averaged over all species $i$. If we follow the normal DID-ESR method of analysis, we would take the log of the left-hand side (lhs) of eq 11, having obtained the tracer concentration profiles at the initial and the final times by DID-ESR. However, in contrast with the single diffusion coefficient case, the plot $\ln [\tilde{C}(\kappa, t) / \tilde{C}(\kappa, 0)]$ vs $\kappa^{2}$ is no longer linear, in principle, and the slope would actually depend on the range of $\kappa^{2}$, as well as on the distribution of species $i$. We can, however, define an effective diffusion coefficient $D_{\text {eff }}$ as

$$
D_{\text {eff }} \equiv-\frac{1}{(2 \pi)^{2} t} \frac{d}{d\left(\kappa^{2}\right)}\left\{\ln \left[\frac{\tilde{C}(\kappa, t)}{\tilde{C}(\kappa, 0)}\right]\right\}
$$

In the limit of a single species (i.e., monodisperse sample), this is easily seen to simplify to $D_{\text {eff }}=D$. In the general case, however, $D_{\text {eff }}$ depends on $\kappa^{2}$. It is rather difficult to derive the physical meaning of $D_{\text {eff }}$ for the case of a polydisperse sample from eq 12. However, we can apply a general approach of probability theory to obtain an approximation to eq 12 . Suppose $F(i)$ is an arbitrary function defined over the $i$ regime and $Y(i)$ is the normalized distribution function for each species $i$, so that we can define an averaged quantity

$$
\langle F(i)\rangle=\int_{i} F(i) Y(i) \mathrm{d} i
$$

The quantity $\ln \langle\exp [F(i)]\rangle$ can be written as a cumulant expansion: ${ }^{9 b}$

$$
\ln \langle\exp [F(i)]\rangle=\sum_{v=1}^{\infty} \frac{H_{v}}{v !}
$$

where $H_{v}$ are the cumulants of the distribution defined as

$$
\begin{aligned}
& H_{1}=\langle F(i)\rangle \\
& H_{2}=\left\langle F(i)^{2}\right\rangle-\langle F(i)\rangle^{2}
\end{aligned}
$$

etc.

For a Gaussian distribution, one has $H_{v}=0$ for $v>2 .{ }^{9}$ The successive cumulants will decrease rapidly in magnitude when $\left|\left(H_{2} / 2\right) / H_{1}\right| \ll 1$. Using the expansion of eq 14 for eq 12 with $F(i)=-(2 \pi \kappa)^{2} t D_{i}$ (cf. eq 11) and keeping only the leading term, we have

$$
D_{\mathrm{eff}}=-\frac{1}{(2 \pi)^{2} t} \frac{d}{d\left(\kappa^{2}\right)}\left\{\ln \left\langle\exp \left[-(2 \pi \kappa)^{2} t D_{i}\right]\right\rangle\right\} \approx\left\langle D_{i}\right\rangle
$$

where $\left\langle D_{i}\right\rangle$ is defined as

$$
\left\langle D_{i}\right\rangle \equiv \int_{i} D_{i} Y(i) \mathrm{d} i
$$

Thus, the traditional DID-ESR analysis would yield the averaged diffusion coefficient, provided the condition

$$
\begin{gathered}
\left|\left\langle F(i)^{2}\right\rangle-\langle F(i)\rangle^{2}\right| / 2\langle F(i)\rangle \ll 1 \\
\text { i.e., } \quad \frac{\left\langle\left(D_{i}-\left\langle D_{i}\right\rangle\right)^{2}\right\rangle}{\left\langle D_{i}\right\rangle^{2}} \ll \frac{2}{(2 \pi \kappa)^{2} t\left\langle D_{i}\right\rangle}
\end{gathered}
$$

is satisfied. In the case of a monodisperse sample, it is always satisfied as one would expect. In the general case, this condition is more readily satisfied when $(2 \pi \kappa)^{2} t\left\langle D_{i}\right\rangle$ is small. In practice, keeping $\kappa^{2}$ and $t$ small reduces the signal to noise ratio, ${ }^{1,2}$ and therefore it is difficult to select an adequate range of $\kappa^{2}$ for a linear fit.

2.2. Nonlinear Least Squares (NLLS) Fit in DID-ESR To Measure $\left\langle D_{i}\right\rangle, \alpha$, and $\boldsymbol{\beta}$ from a Single Polydisperse Polymer Sample in a Single Experiment. In many cases the inequality (19) is not satisfied for polydisperse polymers. For this purpose, we have developed a method utilizing nonlinear least squares (NLLS) fits to eq 11 to determine not only $\left\langle D_{i}\right\rangle$ but also $\alpha$ and $\beta$ from a single experiment on a single sample.

The actual role of polydispersity of the matrix on the diffusion of the tracer molecules is difficult to assess. The easiest limit to consider is that tracer diffusion is relative to a matrix with some averaged molecular weight, $P_{\mathrm{d}}$ (defined in eq 22 below), (to be called case 1). Alternatively, one may wish to model how the tracer diffusion is affected by the actual matrix molecules through which it is diffusing and/or by any preferential diffusion, e.g., tracer molecules seeking out that direction favored by the highest concentration of low molecular weight matrix molecules. Such matters would be very difficult to model. Instead we content ourselves with another limiting case in which there is "full polydispersity"; that is, the observed diffusion of tracer molecules of molecular weight $M_{i}$ is a distribution over the range of matrix molecules with their distribution of molecular weights (to be called case 2). Case 2 undoubtedly exaggerates the effects of matrix polydispersity on polymer diffusion, but it is worth comparing with predictions of case 1 , which most likely oversimplifies their effects. We shall treat case 1 mathematically as a special case of case 2, wherein the polydispersity of matrix molecules is replaced with a monodisperse "average matrix".

We shall use the number-fraction to describe the tracer diffusion and the weight-fraction for the matrix. This is because each tracer molecule is spin labeled by only one free radical moiety, so that the relative intensity of the ESR signal from 
tracer species with molecular weight range $M \rightarrow M+\mathrm{d} M$ is the number-fraction of tracer. (We shall omit the index $i$ of $M, P$ in the following discussion for simplicity.) In cases where the number of labels in the tracer is proportional to the number of monomers instead of polymer molecules, e.g., for perdeuterated tracers used in FRES and NMR, then the signal will be proportional to the weight-fraction of tracer.

We shall assume that the relative contribution from matrix species in the range of $P \rightarrow P+\mathrm{d} P$ is given by its volumefraction, which is essentially the same as the weight-fraction of matrix species, since the volume is proportional to the number of the monomers and their weight.

We now substitute eq 1 into eq 8 to obtain

$$
\left\langle D_{i}\right\rangle=\int_{i} D_{0} M^{-\alpha} P^{-\beta} Y(i) \mathrm{d} i
$$

We shall let $U(M)$ and $V(P)$ be the normalized number-fraction molecular weight distribution (MWD) of tracer and the normalized weight-fraction MWD of matrix, respectively. Substituting these distributions into eq 20 yields

$$
\begin{aligned}
\left\langle D_{i}\right\rangle & =D_{0} \int_{0}^{\infty} M^{-\alpha} U(M) \mathrm{d} M \int_{0}^{\infty} P^{-\beta} V(P) \mathrm{d} P \\
& =D_{0} M_{\mathrm{d}}^{-\alpha} P_{\mathrm{d}}^{-\beta}
\end{aligned}
$$

where we have defined

$$
M_{\mathrm{d}} \equiv\left[\int_{0}^{\infty} M^{-\alpha} U(M) \mathrm{d} M\right]^{-1 / \alpha}, \quad P_{\mathrm{d}} \equiv\left[\int_{0}^{\infty} P^{-\beta} V(P) \mathrm{d} P\right]^{-1 / \beta}
$$

$M_{\mathrm{d}}$ depends upon $\alpha$ and $U(M)$, and $P_{\mathrm{d}}$ upon $\beta$ and $V(P)$. These results apply for case 2 . For case 1 , we let $V(P)=\delta\left(P-P_{\mathrm{d}}\right)$ in eqs 21 and 22. Substituting eq 21 into eq 1 , we have

$$
D_{i}=\left\langle D_{i}\right\rangle\left(M / M_{\mathrm{d}}\right)^{-\alpha}\left(P / P_{\mathrm{d}}\right)^{-\beta}
$$

then the rhs of eq 11 becomes

$$
\begin{aligned}
& \int_{i} \exp \left[-(2 \pi \kappa)^{2} t D_{i}\right] Y(i) \mathrm{d} i= \\
& \int_{0}^{\infty} V(P) \mathrm{d} P \int_{0}^{\infty} U(M) \exp \left[-(2 \pi \kappa)^{2} t\left\langle D_{i}\right\rangle\left(\frac{M}{M_{\mathrm{d}}}\right)^{-\alpha}\left(\frac{P}{P_{\mathrm{d}}}\right)^{-\beta}\right] \mathrm{d} M
\end{aligned}
$$

Next we need to relate the spin concentration profiles on the lhs of eq 11 to the DID-ESR spectra. When applying a linear magnetic field gradient $G$ to a sample with an inhomogeneous spin concentration in the $x$-direction, $C(x, t)$, the magnetic field experienced by spins at position $x$ is spatially dependent according to

$$
B(x)=B_{0}+G x
$$

where $B_{0}$ is the static magnetic field. The gradient-on spectrum, $I_{\mathrm{g}}(B, t)$, is the convolution of the gradient-off spectrum of the spins, $I_{0}(B)$, with the spatial distribution of the spins, $C(B, t)$ :

$$
I_{\mathrm{g}}(B, t)=C(B, t) \otimes I_{0}(B)=\int_{-\infty}^{+\infty} C\left(B^{\prime}, t\right) I_{0}\left(B-B^{\prime}\right) \mathrm{d} B^{\prime}
$$

which becomes after taking Fourier transforms (FTs)

$$
\tilde{I}_{\mathrm{g}}(\xi, t)=\tilde{C}(\xi, t) \tilde{I}_{0}(\xi)
$$

where $\tilde{I}_{\mathrm{g}}(\xi, t), \tilde{C}(\xi, t)$, and $\tilde{I}_{0}(\xi)$ are the FTs of the corresponding functions after converting $B$ into a dimensionless variable, and its FT is $2 \pi \xi$, i.e.,

$$
\left(B-B_{0}\right) / B_{\mathrm{s}}=x G / B_{\mathrm{s}} \leftrightarrow(2 \pi \xi)
$$

where $B_{\mathrm{S}}$ is the sweep range of the ESR spectrum. But eq 11 involves the transformation $x \leftrightarrow(2 \pi \kappa)=2 \pi \xi\left(G / B_{\mathrm{s}}\right)$. Then from eqs 11,24 , and 27 we obtain

$$
\tilde{I}_{\mathrm{g}}(\xi, t)=L(\xi, t) \tilde{I}_{\mathrm{g}}(\xi, 0)
$$

where

$$
\begin{aligned}
L(\xi, t) \equiv \int_{0}^{\infty} V(P) \mathrm{d} P \int_{0}^{\infty} U(M) \times \\
\quad \exp \left[-\left(\frac{2 \pi G \xi}{B_{\mathrm{s}}}\right)^{2} t\left\langle D_{i}\right\rangle\left(\frac{M}{M_{\mathrm{d}}}\right)^{-\alpha}\left(\frac{P}{P_{\mathrm{d}}}\right)^{-\beta}\right] \mathrm{d} M
\end{aligned}
$$

In principle, the three unknown parameters $\left\langle D_{i}\right\rangle, \alpha$, and $\beta$ in eq 30 can be fit by eq 29 from just the two gradient-on ESR spectra obtained when the diffusion process just starts and at a later time. However, this is not practical because of the unsatisfactory signal to noise ratio $(\mathrm{S} / \mathrm{N})$ from just two measurements as well as the difficulty in obtaining an accurate $I_{\mathrm{g}}(B, 0)$ as will be discussed in section 4 . In practice, a set of gradienton spectra are taken at various times $t_{j}(j=1,2, \ldots, J)$ and these $I_{\mathrm{g}}\left(B, t_{j}\right)$ are Fourier transformed to yield $\tilde{I}_{\mathrm{g}}\left(\xi, t_{j}\right)$.

A NLLS fit is used to minimize the deviation between the experimental $\tilde{I}_{\mathrm{g}}\left(\xi, t_{j}\right)$ and its theoretical value, summed over all $t_{j}$ spectra and all $\xi$ points: that is, one varies $\left\langle D_{i}\right\rangle, \alpha$, and $\beta$ to reach the minimum value of the function

$$
\sum_{\xi} \sum_{j}\left[\tilde{I}_{\mathrm{g}}\left(\xi, t_{j}\right)-L\left(\xi, t_{j}\right) \tilde{I}_{\mathrm{g}}(\xi, 0)\right]^{2}
$$

Since, as we noted above, $\tilde{I}_{\mathrm{g}}(\xi, 0)$ is not generally available, and even if it were it would be heavily weighted in the NLLS fit given by eq 31 , we replace it by

$$
\tilde{I}_{\mathrm{g}}(\xi, 0)=\sum_{j=1}^{J} \tilde{I}_{\mathrm{g}}\left(\xi, t_{j}\right) \mid \sum_{j=1}^{J} L\left(\xi, t_{j}\right)
$$

which follows readily from eq 29 .

Finally, for case 1 we shall replace $V(P)$ in eq 30 by $\delta(P-$ $\left.P_{\mathrm{d}}\right)$ to recover

$$
L(\xi, t)=\int_{0}^{\infty} U(M) \exp \left[-\left(\frac{2 \pi G \xi}{B_{\mathrm{s}}}\right)^{2} t\left\langle D_{i}\right\rangle\left(\frac{M}{M_{\mathrm{d}}}\right)^{-\alpha}\right] \mathrm{d} M
$$

Thus $\beta$ may not be determined in this case.

We now use the calculated spectra to illustrate the feasibility of the NLLS fit method. In Figure 3 we plot the FT of gradienton ESR spectra $\tilde{I}_{\mathrm{g}}\left(\xi, t_{j}\right)$ due to different diffusion models and diffusion coefficients, as well as the initial $\tilde{I}_{\mathrm{g}}(\xi, 0)$, from the actual tracer and matrix MWD. Normally, after a certain point $\xi_{\text {max }}$, the magnitude of $\tilde{I}_{\mathrm{g}}\left(\xi, t_{j}\right)$ is within the order of random noise and no longer sensitive to the diffusion process. So in practice, we truncate the $\xi$ range at $\xi_{\max }$, which is about 30 to 40 in our fitting.

With the actual MWD of moderately polydisperse tracer and matrix samples, and by using several sets of $\left\langle D_{i}\right\rangle, \alpha$, and $\beta$ that are close to those observed in our model system $\left(\left\langle D_{i}\right\rangle=10^{-9}\right.$ $\left.10^{-8} \mathrm{~cm}^{2} / \mathrm{s}, \alpha \approx 1.5, \beta=1-2\right)$, we tested the sensitivity and reliability of the NLLS fit method. In the absence of random noise, the accuracy of fit parameters is within $1 \%$ for $\left\langle D_{i}\right\rangle$ and $10 \%$ for $\alpha$ and $\beta$ for a diffusion period of $6.5 \mathrm{~h}$. In the presence of random noise, the uncertainty of $\alpha$ and $\beta$ increases, while the result of $\left\langle D_{i}\right\rangle$ maintains satisfactory accuracy. Under extremely poor $\mathrm{S} / \mathrm{N}$ conditions $(\mathrm{S} / \mathrm{N} \leq 10)$, the output $\left\langle D_{i}\right\rangle$ 
ESR Spectra due to Diffusion Process

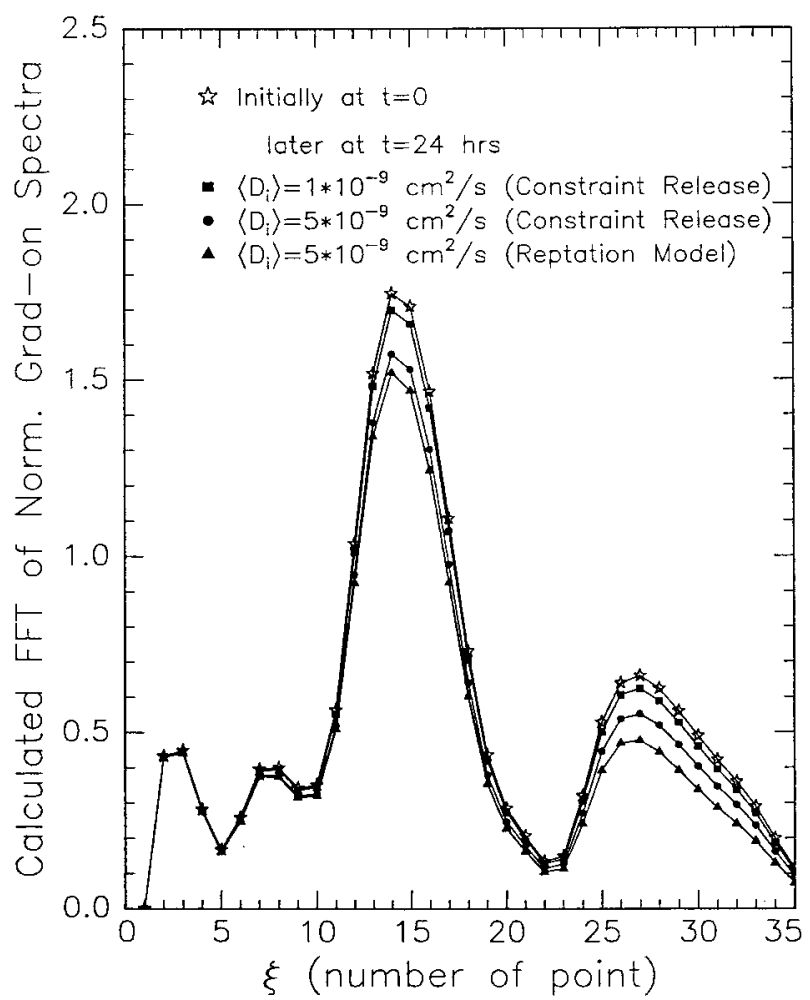

Figure 3. Calculated Fourier transform DID-ESR spectra due to diffusion process from different diffusion coefficients and models: (a) constraint release model, $\left\langle D_{i}\right\rangle=1 \times 10^{-9} \mathrm{~cm}^{2} / \mathrm{s}$ (solid square); (b) constraint release model, $\left\langle D_{i}\right\rangle=5 \times 10^{-9} \mathrm{~cm}^{2} / \mathrm{s}$ (solid circle); (c) reptation model, $\left\langle D_{i}\right\rangle=5 \times 10^{-9} \mathrm{~cm}^{2} / \mathrm{s}$ (solid triangle).

deviates from the input by $10-15 \%$, in contrast to deviations greater than $50 \%$ for $\alpha$ and $\beta$.

It is clear that the NLLS fit is more sensitive to $\left\langle D_{i}\right\rangle$ than $\alpha$ and $\beta$ in these moderately polydisperse samples. Therefore, we average $\left\langle D_{i}\right\rangle$ from several NLLS fit procedures that give comparable least squares from different starting fit parameters and then fix $\left\langle D_{i}\right\rangle$ to further fit $\alpha$ and $\beta$. In this way, under appropriate $\mathrm{S} / \mathrm{N}(\mathrm{S} / \mathrm{N} \approx 50$ in actual experimental spectra) and longer diffusion times (up to $50 \mathrm{~h}$ ), the NLLS fit yields $\left\langle D_{i}\right\rangle=$ $5.08 \times 10^{-9} \mathrm{~cm}^{2} / \mathrm{s}, \alpha=1.55$, and $\beta=1.03$ from the input parameters $\left(\left\langle D_{i}\right\rangle=5 \times 10^{9} \mathrm{~cm}^{2} / \mathrm{s}, \alpha=1.54, \beta=1.93\right)$. Notice the polydispersity is respectively 2.12 and 1.67 for tracer $C$ and matrix $E$ used in the example (cf. Table 1). It is essential to use tracer and matrix samples with large polydispersity to obtain reliable $\alpha$ and $\beta$ values.

2.3. NLLS Fit To Determine $\alpha, \beta$ by $\left\langle D_{i}\right\rangle$ from Several Polydisperse Polymer Samples with Different Tracer and Matrix Molecular Weight Distributions. In the case that reliable values of only $\left\langle D_{i}\right\rangle$ are obtained from a NLLS fit from one sample, we can measure several samples with different tracer and matrix molecular weight distributions under otherwise identical conditions to determine the $\alpha, \beta$ values.

In principle, two samples containing two different tracers but the same matrix are sufficient to determine $\alpha$ from eq 21 . In practice, several samples with different tracers should be utilized to obtain a reliable value of $\alpha$. The NLLS fit method must be applied for such multiple samples.

Considering a set of samples with measured $\left\langle D_{i}\right\rangle_{j}(j=1,2$, ..., $J$ is the sample number), each satisfies eq 21 :

$$
\left\langle D_{i}\right\rangle_{j}=D_{0}\left(M_{\mathrm{d}}\right)_{j}^{-\alpha}\left(P_{\mathrm{d}}\right)_{j}^{-\beta}
$$
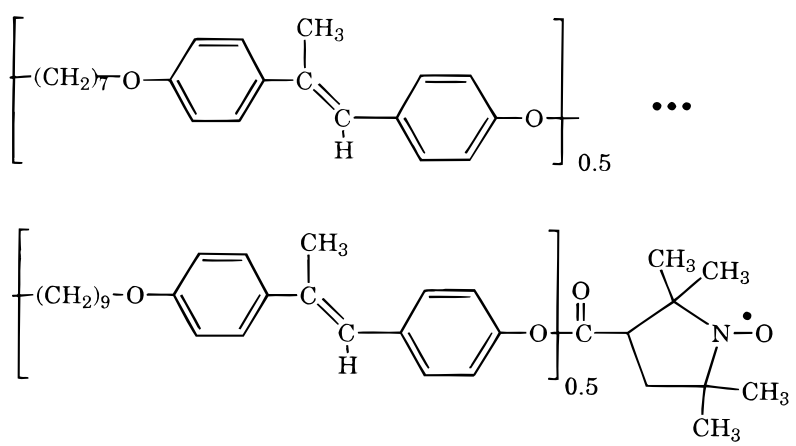

Figure 4. Schematic structure of liquid crystalline random co-poly(ether) DHMS-7,9 end labeled by 3-carboxy-PROXYL.

Then $D_{0}$ can be represented by

$$
D_{0}=\sum_{j=1}^{J}\left\langle D_{i}\right\rangle_{j} \mid \sum_{j=1}^{J}\left(M_{\mathrm{d}}\right)_{j}^{-\alpha}\left(P_{\mathrm{d}}\right)_{j}^{-\beta}
$$

So we utilize NLLS fitting to minimize the summed deviation,

$$
\sum_{k=1}^{J}\left[\left\langle D_{i}\right\rangle_{k}-\left(M_{\mathrm{d}}\right)_{k}^{-\alpha}\left(P_{\mathrm{d}}\right)_{k}^{-\beta} \sum_{j=1}^{J}\left\langle D_{i}\right\rangle_{j} \mid \sum_{j=1}^{J}\left(M_{\mathrm{d}}\right)_{j}^{-\alpha}\left(P_{\mathrm{d}}\right)_{j}^{-\beta}\right]^{2}
$$

to determine $\alpha$ and $\beta$, where each $\left(M_{\mathrm{d}}\right)_{j},\left(P_{\mathrm{d}}\right)_{j}$ is given by the actual molecular weight distribution as defined in eq 22 for case 2 , and they depend on the $\alpha, \beta$ values as well. When only one matrix is used for all samples, it is sufficient to minimize

$$
\sum_{k=1}^{J}\left[\left\langle D_{i}\right\rangle_{k}-\left(M_{\mathrm{d}}\right)_{k}^{-\alpha} \sum_{j=1}^{J}\left\langle D_{i}\right\rangle_{j} \mid \sum_{j=1}^{J}\left(M_{\mathrm{d}}\right)_{j}^{-\alpha}\right]^{2}
$$

in order to obtain $\alpha$. Similarly, $\beta$ can be determined by using the same tracer for all samples.

For case 1 both eqs 36 and 37 also apply. Thus at this stage of the analysis it would not be possible to distinguish between cases 1 and 2 . Note that for case 1, only at this stage would there be any dependence upon $\beta$.

\section{Experiment}

3.1. Materials and Methods. The model system used was a semirigid main chain liquid crystalline poly(ether), based on 4,4'-dihydroxy- $\alpha$-methylstilbene and mixed aliphatic linking groups of 7 and 9 carbons in length (DHMS-7,9). Both the monomer and polymer synthesis have been described previously. ${ }^{10}$ The following procedure describes the attachment of the spin label. The free radical used as a spin label for our ongoing DID-ESR measurements is 3-carboxy-PROXYL (3carboxy-2,2,5,5-tetramethyl-1-pyrrolidinyloxy). The carboxyl functionality of the proxyl molecule was converted to an acid chloride and reacted with the phenolic end groups of the polymer chains. The end-labeled polymer is shown in Figure 4.

The polymer and acid chloride were dried for several days over $\mathrm{CaCl}_{2}$. Anhydrous benzene and pyridine were purchased from Aldrich in Sure-seal bottles and used as received. Chloroform and tetraethylamine (TEA) (both Aldrich) were distilled from $\mathrm{P}_{2} \mathrm{O}_{5}$, and pyridine was distilled from $\mathrm{KOH}$. Thionyl chloride and 3-carboxy-PROXYL (Aldrich) were used as received. The spin probe showed one spot by TLC; the bottle was taken from the refrigerator and warmed to room temperature (RT) in a desiccator to avoid condensation.

A typical reaction is as follows: $1 \mathrm{~g}$ of 3-carboxy-PROXYL (5.37 mmol) was dissolved in $15 \mathrm{~mL}$ of benzene. Using syringe techniques, $540 \mu \mathrm{L}$ of thionyl chloride $(7.4 \mathrm{mmol})$ and 20 drops 


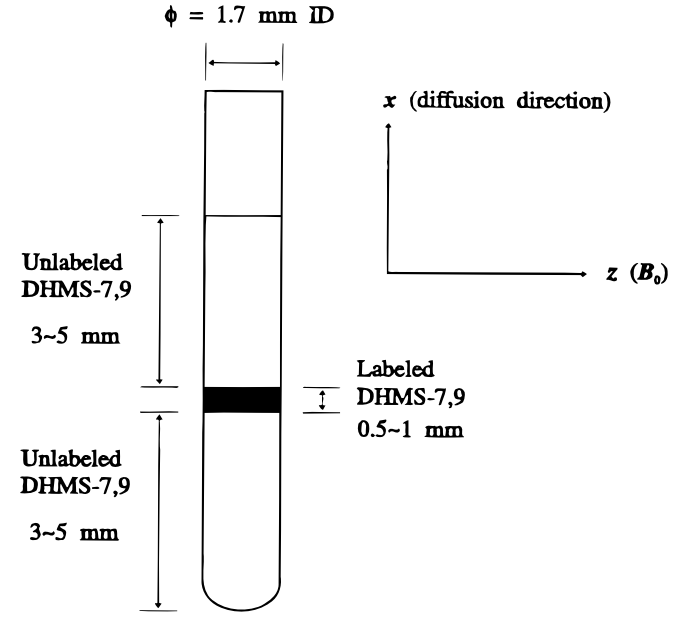

Figure 5. Schematic of the sample for determination of diffusion coefficients in the unrestricted geometry of diffusion by the DID-ESR technique. Note that the magnetic field gradient is along the $x$-direction.

of pyridine $(7.4 \mathrm{mmol})$ were added. The solution was stirred for $1 \mathrm{~h}$ at RT. The crystalline precipitate was filtered off through a sintered filter under $\mathrm{N}_{2}$. The solution was then collected in a $250 \mathrm{~mL}$ 3-neck round bottom flask with a stir bar. The benzene was removed under vacuum at RT.

A $3.8 \mathrm{~g}$ sample of polymer $(1.51 \mathrm{mmol})$ was dissolved in $100 \mathrm{~mL}$ of warm chloroform. The solution was cooled and transferred to the round bottom flask containing the spin probe. The flask was placed in an ice bath and stirred. Ten drops of TEA base was added and the solution allowed to react for $4 \mathrm{~h}$. The polymer was precipitated into methanol. A base extraction of the polymer in a chloroform solution was required to remove the free spin probe, which was not soluble in water or alcohol. The polymer was dried under vacuum at RT and stored under refrigeration.

Differential scanning calorimetry is a sensitive way to check the purity of the spin-labeled samples. A residual amount of free probe showed a very large, narrow exotherm at $200{ }^{\circ} \mathrm{C}$, a temperature higher than the nematic to isotropic transition temperature in these samples. After a base extraction, the narrow exotherm from the free probe disappeared. The purity of the spin-labeled tracer could be confirmed by taking ESR spectra at RT. Any unattached 3-carboxy-PROXYL mixed with unlabeled DHMS-7,9 showed a fast motional component with three sharp hyperfine lines, which was not observed from the spin-labeled DHMS-7,9. Optical microscopy was used to identify the mesophase as nematic.

3.2. DID-ESR Sample Preparation. The samples for the DID-ESR experiment were composed of three layers in a 1.7 $\mathrm{mm}$ i.d. glass tube $(3 \mathrm{~mm}$ o.d.) with a thin layer $(0.5-1 \mathrm{~mm})$ of labeled LC polymer (the tracer) sandwiched between unlabeled LC polymer (the matrix) to form an inhomogeneous distribution of spin concentration in the $x$-direction (perpendicular to the $z$-direction of static magnetic field $\vec{B}_{0}$ ), as shown in Figure 5. The diameter of the tube was selected to be small enough to be considered quasi one-dimensional diffusion yet large enough to avoid significant wall-effects; that is, alignment by surface forces at the tube wall was considered to play a negligible role.

While previously performed DID-ESR experiments measured diffusion constants in a range $10^{-5}-10^{-8} \mathrm{~cm}^{2} / \mathrm{s}$ near RT for ordinary LCs, ${ }^{1-3}$ polymeric LCs have much slower diffusion rates and have relatively high melting temperatures (above 80 ${ }^{\circ} \mathrm{C}$ ). Therefore considerable effort was made to develop a reliable method for sample preparation and to carry out these measurements. The following considerations were particularly relevant.

(1) Spin Concentration. Because the intensity of the ESR signal decreases during the experiment as a result of exposing the free radical to high temperature for several hours, it is very important to start with the highest effective spin concentration in a limited thickness of sample. Above this effective limit, the line shapes of ESR spectra are dependent on concentration, and hence Heisenberg spin exchange (HE) or electron-electron dipolar interactions $(\mathrm{EED})^{11}$ can affect the results. By dissolving a known amount of labeled sample into chloroform and comparing its ESR signal intensity with a perdeuterated Tempone standard in toluene- $d_{8}$ at known concentration, an estimate was made that $60 \%-80 \%$ of the DHMS-7,9 molecules were end labeled. For the tracer $M_{\mathrm{w}}=13400$, the spin concentration was approximately $80 \mathrm{mmol}$ (by assuming the density of DHMS-7,9 melt to be $1 \mathrm{~g} / \mathrm{cm}^{3}$ ) and apparently showed HE and/ or EED effects. Lower molecular weight tracers had higher spin concentration. By means of a concentration dependent study, it was determined that $20 \mathrm{mmol}$ is the maximum effective spin concentration without any $\mathrm{HE}$ and/or EED effects for these samples in their melt. All tracers were diluted to about $20 \mathrm{mmol}$ spin concentration using the appropriate unlabeled matrix.

(2) Thickness of the Tracer Layer and Magnitude of Field Gradient. In principle, a thinner layer of tracer combined with a larger field gradient will reduce the measuring time. However, fewer spins and a broader gradient-on spectrum will significantly reduce the $\mathrm{S} / \mathrm{N}$. An optimum choice of thickness and gradient was found to be $0.5-1 \mathrm{~mm}$ and $G=60 \mathrm{G} / \mathrm{cm}$, yielding an initial line width around $2 \mathrm{G}$ in these particular samples. This combination provided an acceptable $\mathrm{S} / \mathrm{N}$ ratio and a reasonable measuring time (several hours).

(3) Preprocessing the Matrix and Tracer Materials Separately To Make Stock Material Prior To Making the ESR Samples. This is the most critical part of the DID-ESR experiment for LC polymers. During the preprocessing step, samples (matrix or already diluted tracer) were first packed into a $1.7 \mathrm{~mm}$ i.d.) tube as tightly as possible and degassed with a diffusion pump at RT for several hours to remove oxygen. (This dramatically reduced the decomposition rate within the melt.) While still attached to the vacuum system, the sample tube was then put into an oil bath at a temperature higher than the melting point for several minutes, in order to remove more volatile impurities. The sample was degassed at RT again for several hours until a vacuum level $<10^{-4}$ Torr was reached. Next, the tube was put into a heat bath at a temperature high enough to allow the sample to flow down and form a bubble-free stock material.

A thin layer of such preprocessed tracer was sandwiched between the two solid cylindrical pieces of the matrix in a tube of the same size at RT and was slowly lowered into a heat bath (at a rate of approximately $1 \mathrm{~mm} / \mathrm{min}$ ) set at the desired experimental temperature to get any tiny bubbles out without disturbing the initial spin distribution. Nitrogen was blown into the tube during this step and throughout the experiment for deoxygenation. The sample tube was transferred into the ESR cavity for measurement immediately after the whole sample was melted.

3.3. Physical Measurements. Molecular weight distribution functions were determined by a Waters MAXIMA 820 gel permeation chromatography (GPC) instrument using tetrahydrofuran (THF) at $35{ }^{\circ} \mathrm{C}$ as solvent, flow rate $0.3 \mathrm{~mL} / \mathrm{min}$, a $254 \mathrm{~nm}$ UV detector, Ultrastyragel columns of $500,10^{3}$, and $10^{4} \AA$ pore sizes, and a linear column with mixed pore sizes. 
TABLE 1: Molecular Weights and Transition Temperatures of DHMS-7,9 Samples for DID-ESR Measurements ${ }^{a, b}$

\begin{tabular}{crcccccc}
\hline tracer & \multicolumn{1}{c}{$M_{\mathrm{w}}$} & $M_{\mathrm{n}}$ & $M_{\mathrm{w}} / M_{\mathrm{n}}$ & $D_{P_{\mathrm{w}}}$ & $T_{\mathrm{m}}\left({ }^{\circ} \mathrm{C}\right)$ & $T_{\text {xn }}\left({ }^{\circ} \mathrm{C}\right)$ & $T_{\mathrm{ni}}\left({ }^{\circ} \mathrm{C}\right)$ \\
\hline$A$ & 4000 & 3000 & 1.32 & 12 & 92 & & 118 \\
$B$ & 8600 & 4900 & 1.73 & 25 & 96 & 108 & 120 \\
$C$ & 13400 & 6300 & 2.12 & 40 & 82 & & 121 \\
\hline matrix & \multicolumn{1}{c}{$P_{\mathrm{w}}$} & $P_{\mathrm{n}}$ & $P_{\mathrm{w}} / P_{\mathrm{n}}$ & $D_{P_{\mathrm{w}}}$ & $T_{\mathrm{m}}\left({ }^{\circ} \mathrm{C}\right)$ & $T_{\text {xn }}\left({ }^{\circ} \mathrm{C}\right)$ & $T_{\mathrm{ni}}\left({ }^{\circ} \mathrm{C}\right)$ \\
\hline$D$ & 4600 & 3200 & 1.46 & 14 & 90 & & 160 \\
$E$ & 7800 & 4700 & 1.67 & 23 & 102 & 116 & 155 \\
$F$ & 30700 & 18400 & 1.67 & 91 & 91 & 107 & 190
\end{tabular}

${ }^{a} M_{\mathrm{w}}\left(M_{\mathrm{n}}\right)$ are the tracer weight (number) average molecular weights; $P_{\mathrm{w}}\left(P_{\mathrm{n}}\right)$ are the matrix weight (number) average molecular weights; $D_{P_{\mathrm{w}}}$ is the weight average degree of polymerization; $T_{\mathrm{m}}$ is the melting transition temperature; $T_{\mathrm{xn}}$ is the transition temperature from an intermediate phase (exhibited in some materials) to the nematic phase; $T_{\text {ni }}$ is the transition temperature from the nematic to the isotropic phase. ${ }^{b} M_{\mathrm{w}} \equiv \int_{0}^{\infty} M W(M) \mathrm{d} M . \quad M_{\mathrm{n}} \equiv \int_{0}^{\infty} M U(M) \mathrm{d} M . P_{\mathrm{w}} \equiv \int_{0}^{\infty} P V(P) \mathrm{d} P . P_{\mathrm{n}}$ $\equiv\left[\int_{0}^{\infty} P^{-1} V(P) \mathrm{d} P\right]^{-1}$. The relation between $W(M)$ and $U(M)$ is $W(M)$ $=M U(M) / \int_{0}^{\infty} M U(M) \mathrm{d} M$.

Calibration was made with low molecular weight monodisperse polystyrene standards.

Thermal transition temperatures were obtained, and sample purity was checked on a DuPont differential scanning calorimeter interfaced with a DuPont 2000 thermal analysis system and on a Perkin-Elmer DSC-2C differential scanning calorimeter. Heating and cooling rates were $20^{\circ} \mathrm{C} / \mathrm{min}$.

All DID-ESR measurements were performed on a Varian Associates, Inc. (Palo Alto, CA) E-12 spectrometer. The spectra were taken in the first-derivative mode, with a field modulation amplitude about $1 \mathrm{G}$ at $100 \mathrm{kHz}$ modulation frequency. The maximum microwave power without saturation (about $20 \mathrm{~mW}$ ), a large time constant $(0.25 \mathrm{~s})$, and short data acquisition times (1 min) were used for taking the gradient-on spectra to maximize $\mathrm{S} / \mathrm{N}$ without distorting the spectra. The ESR spectra had 100 $\mathrm{G}$ sweep widths. Spectra were digitized to 1024 points and were collected on a 386 PC interfaced to a HP-3457A multimeter. A pair of figure-8 Lewis Coil sets Model 502C from George Associates (Berkeley, CA), driven by a Sorensen DC Power Supply Model SRL20-25, were used to produce a linear field gradient of $63.9 \mathrm{G} / \mathrm{cm}$ at 8.75 A current. A KARON Model 1085 temperature control unit connected to a dewar was used to circulate silicone oil between the cavity and the heat bath to achieve $\pm 1{ }^{\circ} \mathrm{C}$ temperature accuracy. The actual temperature of the samples was measured by a thermocouple inserted into the dewar near the center of the cavity.

\section{Results and Data Analysis}

4.1. The Characterization of Materials. Low molecular weight (MW) fractions of DHMS-7,9 were chosen for two reasons: the first was to achieve melt diffusion coefficients in a range optimal for DID-ESR; the second was to complement the data from previous FRES (forward recoil spectroscopy) studies that focused on the diffusion mechanism over a higher MW range. Data on weight-average molecular weights, numberaverage molecular weights, and transition temperatures are reported in Table 1. But the complete MW distribution functions of the polymer samples are needed for the data analysis. Note that tracer $B$ and matrices $E, F$ (cf. Table 1) exhibit an unusual transition $T_{\mathrm{xn}}$. Studies by X-ray scattering techniques and thermal analysis identify the "intermediate" phase between the melting point $T_{\mathrm{m}}$ and the transition $T_{\mathrm{xn}}$ as a nematic-smectic biphase. ${ }^{12}$ Spin-labeling and sample workup did not change the measured molecular weight of the LC polymer significantly. The combinations formed from three
TABLE 2: DID-ESR Samples Composed by Tracers and Matrices

\begin{tabular}{cccc}
\hline & \multicolumn{3}{c}{$\operatorname{tracer}^{a}$} \\
\cline { 2 - 4 } matrix & $A$ & $B$ & $C$ \\
\hline$D$ & $A D$ & $B D$ & \\
$E$ & $A E$ & $B E$ & $C E$ \\
$F$ & $A F$ & $B F$ &
\end{tabular}

${ }^{a}$ Tracers in DID-ESR samples were first diluted by the appropriate matrices to reduce the spin concentration. For example, sample $A D$ consisted of labeled layer of tracer $A$ diluted by matrix $D$ and sample $B D$ consisted of labeled layer of tracer $B$ diluted by matrix $D$, etc. The tracer information listed in Table 1 is for the material before dilution.

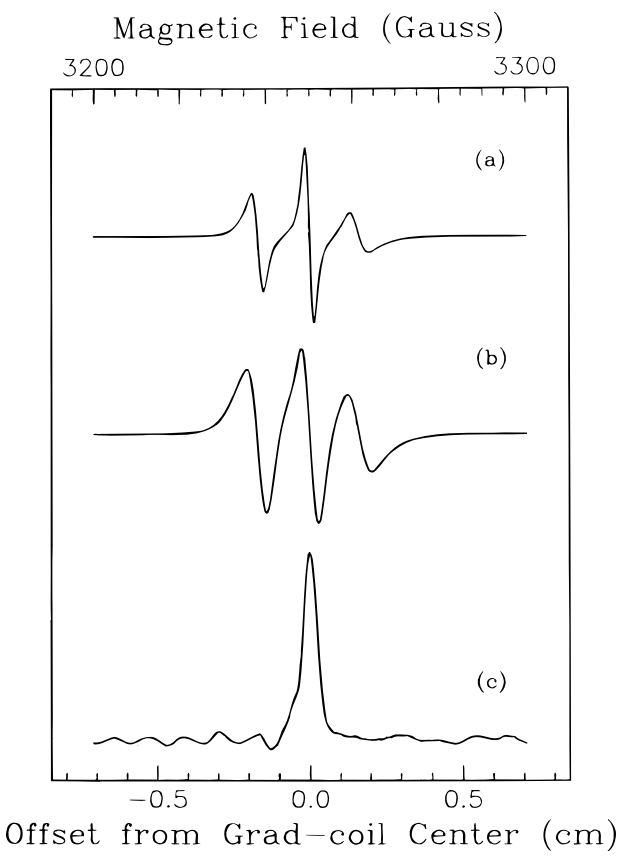

Figure 6. Initial spin concentration profile of sample $A D$ by the deconvolution method: (a) gradient-off ESR spectrum; (b) gradienton ESR spectrum; (c) initial spatial distribution of tracer that was obtained by deconvolution of $\mathrm{b}$ from $\mathrm{a}$.

tracers and three matrices yielded seven useful DID-ESR samples (cf. Table 2). A temperature of $120^{\circ} \mathrm{C}$ was chosen to measure the diffusion coefficient, because it is in the nematic regime of the matrices, and spin intensity does not decay significantly at this temperature. It is close to the temperature of the nematic to isotropic transition $\left(T_{\mathrm{ni}}\right)$ of all three tracers.

4.2. Initial Spin Concentration Profile. At the beginning of each DID-ESR measurement, the field gradient-off and gradient-on spectra were taken so that we could use the deconvolution method to quickly obtain the initial spin concentration profile. In this way, we could check the quality of the DID-ESR sample preparation and therefore estimate the time needed for each measurement. Typical results are presented in Figure 6. In early stages of the development of the DID-ESR technique for fast diffusion $\left(\sim 10^{-5} \mathrm{~cm}^{2} / \mathrm{s}\right)$, the deconvolution method was used to yield the spin concentration profile at the initial and final times and thus the diffusion coefficient. However, this method requires one to perform the inverse Fourier transform, which results in additional numerical errors. ${ }^{13}$ In the present DID-ESR experiment, we calculate diffusion coefficients in the Fourier domain, as discussed in section 2 and shown below.

4.3. Macroscopic Alignment of Polymeric LC Melts by ESR Field. An interesting phenomenon was observed during the DID-ESR experiments, namely, macroscopic alignment at the rather low magnetic fields used. In some of the LC 
polymers, alignment was occurring in the nematic phase in a magnetic field of about $3250 \mathrm{G}$, a field not normally strong enough to align main chain LC polymer samples. Initially, the directors of local domains of the polymer molecules were randomly distributed, while local order exists within each domain. Such a state can be described by a MOMD (microscopic order macroscopic disorder) model. ${ }^{14,15}$ However, we found that the splitting of the ESR peaks, characteristic of MOMD, were decreasing as the time increased. This reduction of peak splittings indicates that the spin labels were taking on a preferred direction due to the magnetic field. This clearly implies that the LC polymer molecules were being macroscopically aligned and the directors of all the local domains were becoming parallel to the magnetic field direction $\vec{B}_{0}$ (z-axis), giving ESR spectra with a smaller splitting than observed for isotropic fluids. ${ }^{15}$ After the alignment process was complete, the gradient-off ESR spectra remained unchanged and the associated gradient-on ESR spectra were used to calculate the diffusion coefficients according to section 4.4. To further confirm our observations, gradient-off ESR spectra were taken again after the DID-ESR measurements. For those macroscopically aligned samples, the ESR spectra showed significant orientation dependence, such that the splittings in the case of $\vec{d} \perp \vec{B}_{0}$ was greater than that the $\vec{d} \| \vec{B}_{0}$ case and the initial MOMD case.

For those samples that remained macroscopically unaligned (MOMD), their gradient-off ESR spectra did not change during the DID-ESR experiment of several hours. They yield spectra that are unchanged by rotating the sample tube along the $x$-axis. The degree of alignment depends largely on the matrix rather than on the tracer. While matrices of smaller MW $\left(P_{\mathrm{w}}=4600\right)$ could be well aligned within $1 \mathrm{~h}$ (samples $A D$ and $B D$ ), the larger MW matrix $\left(P_{\mathrm{w}}=30700\right.$, samples $A F$ and $\left.B F\right)$ showed only partial alignment after $20 \mathrm{~h}$, and the ESR spectra consisted of superpositions of aligned and MOMD components. This lineshape pattern of two superposed components was only observed in the samples using matrix $F$, strongly suggesting that the ESR signal from the tracer reflects the alignment of the matrix. At this stage, the absence of any alignment in samples $C E$, in contrast to samples $A D, B D, A E$, and $B E$, appears to be due to sample preparation. Due to a limited amount of material, duplicate samples could not be tested. Instead, we compared the behavior of the different samples to estimate the reliability of the results. The gradient-off spectra of these DID-ESR samples were analyzed by simulation methods to yield the ordering parameter and the rotational diffusion coefficients. ${ }^{15}$

The details of the simulations can be found in ref 15 , and we simply provide the orientational order parameter $S$ of these samples in Table 3 . Since $S=1$ indicates complete alignment with respect to the nematic director, while $S=0$ indicates no alignment, we can generalize from the measured values that the end-labeled sites of the polymers are only moderately aligned along the director. ${ }^{16}$ The effect of alignment on the diffusion coefficient measurement will be discussed in section 5 .

4.4. Translational Diffusion Coefficients. The translation diffusion coefficient $\left\langle D_{i}\right\rangle$ of each sample was determined by the NLLS analysis described in section 2.2, from the gradienton ESR spectra obtained after the gradient-off ESR spectra became time independent, showing that the alignment process was completed. We have assumed that for each species in the polydisperse sample the alignment effects are the same and yield the same degree of ordering for all species. The influence of ordering on the measured diffusion coefficient will be discussed in section 5.1 .

We averaged each spectrum 10 times to improve $\mathrm{S} / \mathrm{N}$, and we normally took $10-15$ such averaged spectra covering the
TABLE 3: Translational Diffusion Coefficients of DHMS-7,9

\begin{tabular}{|c|c|c|c|c|c|c|}
\hline sample $^{a}$ & $\begin{array}{l}\left\langle D_{i}\right\rangle_{\exp }{ }^{b} \\
\left(\times 10^{9}\right) \\
\left(\mathrm{cm}^{2} / \mathrm{s}\right)\end{array}$ & $S^{c}$ & $\begin{array}{c}\bar{D}_{i, \text { extrap }}{ }^{d} \\
\left(\times 10^{10}\right) \\
\left(\mathrm{cm}^{2} / \mathrm{s}\right)\end{array}$ & $\begin{array}{l}\left\langle D_{i}\right\rangle_{\text {est }}{ }^{e} \\
\left(\times 10^{9}\right) \\
\left(\mathrm{cm}^{2} / \mathrm{s}\right)\end{array}$ & $\begin{array}{c}\bar{D}_{i, \text { extrap }}^{f} \\
\left(\times 10^{10}\right) \\
\left(\mathrm{cm}^{2} / \mathrm{s}\right)\end{array}$ & $\begin{array}{l}\left\langle\left\langle D_{i}\right\rangle_{\mathrm{est}}{ }^{e}\right. \\
\left(\times 10^{9}\right) \\
\left(\mathrm{cm}^{2} / \mathrm{s}\right)\end{array}$ \\
\hline$A D^{*}$ & 110 & 0.42 & 11 & 6.3 & 19 & 10.9 \\
\hline$B D^{*}$ & 15 & 0.36 & 5.0 & 4.0 & 7.0 & 5.6 \\
\hline$A E^{*}$ & 16 & 0.32 & 3.2 & 2.9 & 6.6 & 6.0 \\
\hline$B E^{*}$ & 6.7 & 0.26 & 1.5 & 1.8 & 2.5 & 3.0 \\
\hline$C E^{+}$ & 3.1 & & 0.94 & 1.7 & 1.4 & 2.5 \\
\hline$A F^{\#}$ & 7.6 & 0.26 & 0.14 & 0.20 & 0.51 & 0.73 \\
\hline$B F^{\#}$ & 1.7 & 0.26 & 0.063 & 0.12 & 0.19 & 0.36 \\
\hline
\end{tabular}

$a *$ indicates a macroscopically aligned sample; + indicates a MOMD sample; \# indicates a mixed sample. ${ }^{b}\left\langle D_{i}\right\rangle_{\exp }$ is determined by the NLLS fit DID-ESR. The estimated error is $\pm 10 \% .{ }^{c} S$ is the orientational order parameter from ref 15 . The estimated deviation is $\pm 0.01{ }^{d} \bar{D}_{i, \text { extrap }}$ was determined by using the FRES diffusion results from ref $6\left(M_{\mathrm{w}}=\right.$ $29000, P_{\mathrm{w}}=15500, D_{i}=9 \times 10^{-12} \mathrm{~cm}^{2} / \mathrm{s}$ ) and eq $42 .{ }^{e}\left\langle D_{i}\right\rangle_{\mathrm{est}}$ was calculated by using the entries for $\bar{D}_{i, \text { extrap }}$ in the previous column, eq 41 and the "diffusion average" molecular weights listed in Table 4. ${ }^{f} \bar{D}_{i, \text { extrap }}$ was determined by using the FRES diffusion result from ref 6 and the values of $\alpha=-1.5$ and $\beta=-1.9$ obtained in the present study.

entire measuring period (several hours) for the NLLS fit. These spectra were first integrated and normalized to the total spin number to correct for the signal decay, assuming that the spin decay rate is independent of the MW of the individual diffusion species.

The normalized spectra were then Fourier transformed to yield the $\tilde{I}_{\mathrm{g}}\left(\xi, t_{j}\right)$ (cf. eq 27 ), which were simultaneously fit by the NLLS fitting process associated with eqs 31 and 32 using the principal axis method. ${ }^{17}$ With several sets of different starting values for $\left\langle D_{i}\right\rangle, \alpha$, and $\beta$, the NLLS fit was iterated to yield a minimum deviation, normally corresponding to several different combinations of $\left\langle D_{i}\right\rangle, \alpha$, and $\beta$ values. Due to insufficiently large polydispersities of tracer and matrix polymers and to the limited time range (several hours), the fitting was much more sensitive to $\left\langle D_{i}\right\rangle$ than to $\alpha$ and $\beta$. The deviation of $\left\langle D_{i}\right\rangle$ from the several outputs was usually less than $10 \%$, while no consistent $\alpha, \beta$ values were obtained due to their much larger deviations. In Figure 7 , we plot the experimental $\tilde{I}_{\mathrm{g}}\left(\xi, t_{j}\right)$ and the corresponding outputs from the NLLS fit of sample $A E$. The $\left\langle D_{i}\right\rangle$ values of all seven samples are listed in Table 3.

4.5. Molecular Weight Dependence of Diffusion Coefficient. Because no reliable values of $\alpha, \beta$ were obtained from a single sample, we needed to apply the NLLS fit method of section 2.3 to determine their values. However, this approach requires all samples to be measured under identical conditions, as can be seen from the derivation, and the reliability of the results is subject to the accuracy of the measured $\left\langle D_{i}\right\rangle$. Considering the alignment effect described in section 4.3 and the deviation of each $\left\langle D_{i}\right\rangle$, we chose samples $A E, B E$, and $C E$ to fit $\alpha$, and samples $B D, B E$, and $B F$ to fit $\beta$, respectively. These two groups consist of the maximum number of samples of the same matrix (tracer) and with relatively small differences in their order parameter (cf. Table 3), so that its influence can be minimized. In this way, we get $\alpha=1.5$ and $\beta=1.9$. This result and its comparison with previous FRES results will be addressed in section 5 .

\section{Discussion}

5.1. Comparison between the DID-ESR and FRES Results To Study the MW Dependence of Diffusion. To compare the DID-ESR results with those from FRES on this model LC polymer system, it is important to consider three key differences. First, in a polydisperse sample the effective or "average" diffusion coefficient that is observed, because of a 
Nonlinear Least Squares Fit to Data Set

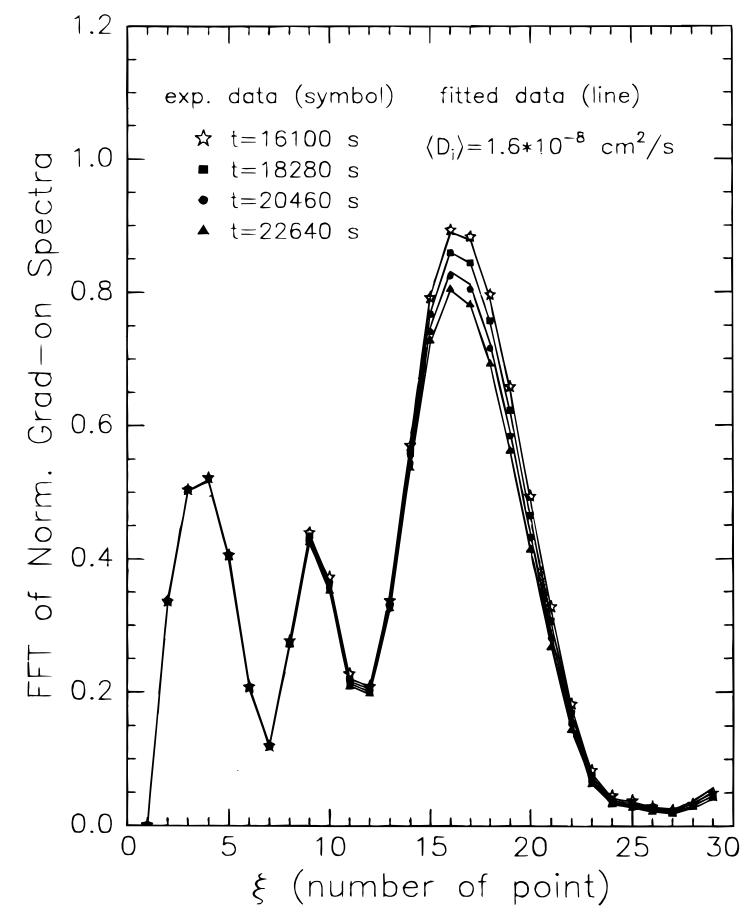

Figure 7. Nonlinear least squares fitting (solid lines) to Fourier transforms (symbols) of the DID-ESR spectra of sample, $A E$, which yields $\left\langle D_{i}\right\rangle=1.6 \times 10^{-8} \mathrm{~cm}^{2} / \mathrm{s}$.

different weighting over the sample's MW distribution, is different in the two experiments. Second, the FRES studies were performed on tracer polymer molecules that were significantly higher in molecular weight than those used in this DIDESR study, and this was also generally true for the matrix. Third, we must consider the differences in preparation and alignment of the DID-ESR samples vs the FRES samples, which can affect the observed diffusion coefficients. We first consider the different average diffusion coefficient obtained by FRES.

The translational diffusion coefficient measured by the FRES technique, $\overline{D_{i}}$, in principle corresponds to a FRES associated "diffusion average molecular weight", $M_{\mathrm{D}}$ and $P_{\mathrm{D}}$, for the tracer and the matrix, respectively. ${ }^{6,18-21}$ Their definitions are given by

$$
\overline{D_{i}} \equiv\left(\int_{i} D_{i}^{-1 / 2} Y(i) \mathrm{d} i\right)^{-2}=D_{0} M_{\mathrm{D}}^{-\alpha} P_{\mathrm{D}}^{-\beta}
$$

where $M_{\mathrm{D}}$ and $P_{\mathrm{D}}$ are defined as

$$
\begin{gathered}
M_{\mathrm{D}} \equiv\left[\int_{0}^{\infty} M^{\alpha / 2} W(M) \mathrm{d} M\right]^{2 / \alpha} \\
P_{\mathrm{D}} \equiv\left[\int_{0}^{\infty} P^{\beta / 2} V(P) \mathrm{d} P\right]^{2 / \beta}
\end{gathered}
$$

and $W(M)$ is the normalized weight-fraction MWD of tracer (cf. caption to Table 1). This is because the FRES signal is proportional to the number of monomers in the tracer molecule (cf. section 2.2 and refs 6, 18-21). When both the tracer and the matrix MW distributions are taken into account, the diffusion coefficients measured by DID-ESR and FRES of an identical sample under identical conditions (temperature, ordering, etc.) is related by

$$
\frac{\left\langle D_{i}\right\rangle}{\overline{D_{i}}}=\frac{M_{\mathrm{d}}^{-\alpha}}{M_{\mathrm{D}}^{-\alpha}} \frac{P_{\mathrm{d}}^{-\beta}}{P_{\mathrm{D}}^{-\beta}}
$$

TABLE 4: "Diffusion Average" Molecular Weights of DHMS-7, ${ }^{a}(\alpha=1, \beta=2.3)$

\begin{tabular}{cccccc}
\hline tracer & $M_{\mathrm{d}}$ & \multicolumn{1}{c}{$M_{\mathrm{D}}$} & $\left(M_{\mathrm{d}} / M_{\mathrm{D}}\right)^{-1}$ & $M_{\mathrm{D}} / M_{\mathrm{w}}$ & $M_{\mathrm{d}} / M_{\mathrm{w}}$ \\
\hline$A$ & 2500 & 3700 & 1.48 & 0.92 & 0.62 \\
$B$ & 3600 & 7100 & 1.98 & 0.84 & 0.42 \\
$C$ & 3600 & 10700 & 2.95 & 0.80 & 0.27 \\
\hline matrix & $P_{\mathrm{d}}$ & \multicolumn{1}{c}{$P_{\mathrm{D}}$} & $\left(P_{\mathrm{d}} / P_{\mathrm{D}}\right)^{-2.3}$ & $P_{\mathrm{D}} / P_{\mathrm{w}}$ & $P_{\mathrm{d}} / P_{\mathrm{w}}$ \\
\hline$D$ & 2600 & 4700 & 4.01 & 1.03 & 0.56 \\
$E$ & 3800 & 8200 & 6.02 & 1.05 & 0.48 \\
$F$ & 11900 & 31800 & 9.52 & 1.04 & 0.39
\end{tabular}

${ }^{a}$ The diffusion-average MWs are represented by the subscripts $\mathrm{d}$ (for DID-ESR) and D (for FRES), with w representing the weightaverage MWs. The MWs of the tracer and the matrix are represented by the letters $\mathrm{M}$ and $\mathrm{P}$, respectively. The values of $\alpha, \beta$ from the FRES experiments are used to calculate $M_{\mathrm{d}}, M_{\mathrm{D}}, P_{\mathrm{d}}$, and $P_{\mathrm{D}}$. The values of $M_{\mathrm{w}}$ and $P_{\mathrm{w}}$ are listed in Table 1.

This ratio ranges from 6 to 30 for the polydisperse samples that we use (cf. Table 4).

Secondly, the FRES experiments measured $\overline{D_{i}}$ for DHMS7,9 of higher MWs and showed that its dependence on the molecular weights of matrix and tracer is given by 6,21

$$
\overline{D_{i}} \tilde{\propto} M_{D}^{-1} P_{D}^{-2,3}
$$

(where we have used the fact ${ }^{18-21}$ that $M_{\mathrm{w}} \approx M_{\mathrm{D}}$, which is also appropriate for the present samples, cf. Table 4). Given the experimental uncertainties, the exponents from FRES in eq 43 are not substantially different from the values of $\alpha=-1.5$ and $\beta=-1.9$ in the present study. Now let us use eq 42 to extrapolate from the results of the higher MW FRES experiment to predict values $\bar{D}_{i \text {,extrap }}$ for the MWs studied by DID-ESR. We obtain the entries in Table 3 (fourth column). We find that these results range from 30 to 500 times smaller than $\left\langle D_{i}\right\rangle$ in column 2 of Table 3 . When we next correct the results in Table 3 , column 4 for the experimental differences between DID-ESR and FRES expressed by eq 41 , we obtain the estimates given in column 5. These estimates of $\left\langle D_{i}\right\rangle$ are typically $2-5$ times smaller than the experimentally measured values in column 2 for the macroscopically aligned and MOMD samples (except for sample $A D$, where it is 17 times smaller). They are also 15-40 times smaller for the two results in the largest MW matrix. Given the nature of the various approximations, this is probably not an unreasonable comparison. Certainly, the extrapolation of eq 42 can be questioned in its accuracy and in the applicability of the exponents from FRES when applied to the lower MW LC polymers. In fact, if we use the exponents of $\alpha=-1.5$ and $\beta=-1.9$, obtained in our present study, to extrapolate from the higher MW FRES experiment, we obtain a significantly better comparison between the predicted results (cf. Table 3, columns 5 and 6). These estimates (i.e. $\left\langle D_{i}\right\rangle_{\mathrm{est}}$ ) are typically $1.3-3$ times smaller than the experimental values in column 2 for the aligned and MOMD samples (with sample $A D$ now 10 times smaller). An even larger improvement in the comparison is obtained for the two results in the largest MW matrix: the estimates are now only 5-10 times smaller.

When we consider the respective sample preparations and their alignment, it seems reasonable to expect that the experimental values of $\left\langle D_{i}\right\rangle$ would be larger than those estimated from the previous FRES experiments. In the FRES experiments, the $\bar{D}_{i}$ were measured in nearly perfectly aligned films (estimated order parameter, $S \geq 0.9$ ), with the diffusion occurring perpendicular to the direction of alignment, i.e. $\bar{D}_{i} \approx\left(\bar{D}_{\perp}\right)_{i}$. It has been previously pointed out ${ }^{22}$ that the LC polymer chains, when extended in a nematic melt, will have a far greater axial 
ratio than monomer liquid crystals, resulting in a large morphological anisotropy, which can be expected to yield a diffusion tensor with a $D_{\|}$(i.e. diffusion coefficient for motion parallel to the direction of alignment) significantly larger than $D_{\perp}$. In the present DID-ESR study the magnetic field aligns the nematic director parallel to the field, and this is perpendicular to the direction of diffusion. Thus, although our experimental arrangement nominally measured $\left\langle\left(D_{\perp}\right)_{i}\right\rangle$ in our macroscopically aligned samples, these samples are not as well aligned, as judged by the values of $S$ in Table 3, while the samples $A F$ and $B F$ are a mixture of aligned and misaligned regions. For LCs that are partially aligned, it is well-known that the measured $D_{\perp}$ is an admixture of $D_{\perp}^{0}$ and $D_{\|}^{0}$, which are the perpendicular and parallel components, respectively, of the diffusion tensor for a perfectly aligned sample. ${ }^{23}$ Thus, since one expects $D_{\|}^{0}$ $\gg D_{\perp}^{0}$, as we have just noted, an admixture of $D_{\|}^{0}$ into the measured $D_{\perp}$ will increase its magnitude, and the effect should be larger in the poorly aligned samples, which in our study includes those with the largest MW matrix (i.e. samples $A F$ and $B F) .^{24}$

5.2. Proposed Future Experiments To Study the Matrix Effect in the LC Polymer Diffusion Mechanism. A more complete study including the MW dependence of the DID-ESR average for both $D_{\perp}$ and $D_{\|}$, i.e. $\left\langle\left(D_{\perp}\right)_{i}\right\rangle$ and $\left\langle\left(D_{\|}\right)_{i}\right\rangle$, as well as the details of the matrix effect in the diffusion process, would require more experiments in which all diffusion samples can be aligned by a very high magnetic field (up to $9 \mathrm{~T}$ ) to achieve uniform macroscopic ordering that will not be largely altered by an X-band ESR magnetic field (as we have demonstrated in a preliminary experiment). Then by properly orienting the sample, one can measure both $\left\langle\left(D_{\perp}\right)_{i}\right\rangle$ and $\left\langle\left(D_{\|}\right)_{i}\right\rangle .{ }^{1-4}$

The ideal situation would be a successful measurement on several samples with large polydisperse tracer and matrix MW distributions, so that each sample can readily yield reliable $\left\langle D_{i}\right\rangle$, $\alpha$, and $\beta$ from NLLS fits. The advantage of obtaining $\alpha$ and $\beta$ from a single sample is that one can eliminate any factors in the preparation (e.g. degree of alignment) that may affect $D_{0}$ in the different samples (cf. section 2.2).

If $\beta=0$ is consistently obtained from each sample (e.g. case 1 of section 2.2), one needs to use the method of section 2.3 to fit the $\beta$ value (this requires that all samples be measured under the same conditions except for different matrix MWs), in order to determine whether the matrix actually exhibits no effect on the tracer diffusion or its effect can be described by an average MW (which is case 1 in section 2.2).

Given that DID-ESR and FRES measure different average diffusion coefficients, additional insight into the role of the matrix could be obtained from measurements on the same type of sample by both methods. This would be most appropriate provided that a nonzero $\beta$ is obtained in the measurements. It would be of greatest interest if $\beta \neq 0$ is obtained from each sample but no consistent $\beta$ is obtained from a set of samples, since this would imply that the limiting case 2 of "full polydispersity" (cf. section 2.2) is not fully applicable. On the other hand, if $\beta=0$ is obtained from each sample, implying case 1 , then the ratio of diffusion coefficients measured by DIDESR and FRES would be independent of the matrix, which can be verified by using the same tracer and different matrices for both experiments.

Additionally, it would be of value to perform the DID-ESR experiments on a polymer system for which no complications are expected from ordering of the matrix in the magnetic field in order to further clarify its applications to polydisperse systems.

\section{Summary}

In summary, we have demonstrated that in the presence of polydispersity with respect to the molecular weights, the polymer samples should exhibit a quite large distribution of diffusion coefficients, whose measurement in general would be different from the traditional method for a single diffusion coefficient. ${ }^{25}$ For this reason, we have developed a new data analysis procedure utilizing a nonlinear least squares (NLLS) fit to measure an average diffusion coefficient by DID-ESR and for samples with large polydispersity a simultaneous determination of the tracer and matrix molecular weight dependence of diffusion coefficient as well. The reliability and sensitivity of this methodology was discussed by theoretical simulation of data sets. We also discussed the conditions under which the average diffusion coefficient can be approximated as a monodisperse diffusion case using the traditional DID-ESR data analysis. The principles applied in the DID-ESR technique are also valid for other techniques for accurate measurement of diffusion coefficients in a polydisperse polymer system.

The melt diffusion in a liquid crystalline polymer system was studied by NLLS fitting of the DID-ESR experiment. The results were compared with those from previous FRES experiments. After considering the differences in these two techniques, the MWs of the respective samples, and differences in their preparation and alignment, it was shown that the results obtained by the two methods are reasonably consistent. It was also shown that the diffusion in these samples depends on both matrix and tracer molecular weights, consistent with the FRES study. The measurement of rotational diffusion is discussed in a separate paper. ${ }^{15}$

Acknowledgment. The authors would like to thank Dr. R. H. Crepeau for his help in preparation of the manuscript and Dr. S. G. McNamee for his help with the X-ray diffraction experiments. This work was supported by the MRL Program of the National Science Foundation under award No. DMR9121654 and by NSF Grants No. DMR-9210638 and CHE-9313167. The Cornell Theory Center and the Cornell Materials Science Center are also acknowledged.

\section{References and Notes}

(1) Moscicki, J. K.; Shin, Y.-K.; Freed, J. H. In EPR Imaging and In Vivo EPR; Eaton, G. F., Eaton, S. S., Ohno, K., Eds.; CRC Press: Boca Raton, 1991; Chapter 19.

(2) Moscicki, J. K.; Shin, Y.-K.; Freed, J. H. (a) J. Magn. Reson. 1989 84, 554; (b) J. Chem. Phys. 1993, 99, 634. (c) Shin, Y.-K.; Moscicki, J. K.; Freed, J. H. Biophys. J. 1990, 57, 445.

(3) Freed, J. H. Annu. Rev. Biophys. Biomol. Struct. 1994, 23, 1.

(4) Freed, J. H.; Nayeem, A.; Rananavare, S. B. In The Molecular Dynamics of Liquid Crystals; Luckhurst, G. R., Veracini, C. A., Eds.; Kluwer: Netherlands, 1994; Chapter 12.

(5) (a) Doi, M.; Edwards, S. F. The Theory of Polymer Dynamics; Clarendon Press: Oxford, New York, 1986. (b) Szleifer, I.; Wilson, J. D.; Loring, R. F. J. Chem. Phys. 1991, 95, 8474.

(6) Hall, E.; Ober, C. K.; Kramer, E. J.; Colby, R. H.; Gillmor, J. R. Macromolecules 1993, 26, 3764.

(7) Schlick and co-workers [Schlick, S.; Pilar, J.; Kweon, S.-C.; Vacik, J.; Gao, Z.; Labsky, J. Macromolecules 1995, 28, 5780] have employed spectral-spatial ESR imaging to study diffusion of small molecular weight molecules in polymers. Theirs is a case of monodisperse diffusion.

(8) A preliminary account of this work was presented at the ACS meeting March, 1994, San Diego [Xu, D.; Budil, D. E.; Freed, J. H.; Hall, E.; Ober, C. K. Polym. Prepr. 1994, 35, 809]. It did not consider the polydispersity of the polymer samples.

(9) (a) Crank, J. The Mathematics of Diffusion; Clarendon Press: Oxford, 1975. (b) Van Kampen, N. G. Stochastic Processes in Physics and Chemistry; North-Holland Publishing Co.: Amsterdam, 1981.

(10) Angelopoulos, E. H.; Ober, C. K.; Kramer, E. J. Proc. ACS Div. Polym. Mat.: Sci. Eng. 1990, 63, 450.

(11) Nayeem, A.; Rananavare, S. B.; Sastry, V. S. S.; Freed, J. H. J. Chem. Phys. 1989, 91, 6887. 
(12) Hall, E.; Robinson, A. A.; McNamee, S. G.; Ober, C. K.; Freidzon, Y. S. J. Mater. Sci. 1995, 30, 2023.

(13) Cleary, D. A.; Shin, Y.-K.; Schneider, D. J.; Freed, J. H. J. Magn. Reson. 1988, 79, 474.

(14) Meirovitch, E.; Nayeem, A.; Freed, J. H. J. Phys. Chem. 1984, 88 (16), 3454.

(15) Xu, D.; Budil, D. E.; Ober, C. K.; Freed, J. H. J. Phys. Chem. 1996, $100,15867$.

(16) We attempted to confirm these measured $S$ values with X-ray diffraction measurements of the solid samples, which showed weakly orientational dependent ESR spectra at room temperature. Statton camera experiments using a $\mathrm{Cu} \mathrm{K \alpha}$ source and flat film exposed for $8 \mathrm{~h}$ failed to show any discernible alignment. We believe this may be due to the lower sensitivity of the X-ray technique or perhaps to a partial loss of order during crystallization after the DID-ESR experiment.

(17) Brent, R. P. Algorithms for Minimization without Derivatives; Prentice-Hall, Inc.: Englewood Cliffs, NJ, 1973.

(18) Klein, J.; Briscoe, B. J. Proc. R. Soc. London A 1979, 365, 53.

(19) Mills, P. J.; Green, P. F.; Palmstrom, C. J.; Mayer, J. W.; Kramer, E. J. Appl. Phys. Lett. 1984, 45, 957.

(20) Mills, P. J.; Green, P. F.; Palmstrom, C. J.; Mayer, J. W.; Kramer, E. J. J. Polym. Sci.: Polym. Phys. Ed. 1986, 24, 1.
(21) Xu, D. Ph.D. Thesis, Cornell University, 1996

(22) Hall, E.; Ober, C. K.; Galli, G. Liq. Cryst. 1993, 14 (5), 1351.

(23) Kruger, G. J. Phys. Rep. 1982, 82 (4), 229, and references therein.

(24) We do not explicitly try to estimate $D_{\perp}^{0}$ and $D_{\|}^{0}$ from the combined FRES and DID-ESR data with the values of $S$ in Table 3 and the wellknown equations, ${ }^{21,23}$ because (1) these values of $S$ depend on both the overall molecular alignment, which is the important quantity, and the ordering of the labeled moiety relative to the molecular backbone; we are not able to separate the two here, but see: Xu, D,; Crepeau, R. H.; Ober, C. K.; Freed, J. H. J. Phys. Chem. 1996, 100, 15873. Also, (2) the existing theory ${ }^{23}$ simply includes the geometric factors appropriate when observations are made on partially aligned liquid crystalline systems. It does not allow for the possibility that the degree of macroscopic alignment has an effect on the microscopic structure, hence on the diffusion process.

(25) Chen, A.; Wu, C.; Johnson, C. S., Jr. [J. Am. Chem. Soc. 1995, $117,7965]$ have considered the effect of polydispersity in NMR experiments on diffusion in polyethylene. In that case monodisperse samples could first be studied to obtain $D_{0}, \alpha$, and $\beta$. Then an analysis of the diffusional polydispersity in commercial samples could be used to obtain their molecular weight distributions.

JP960513L 